\title{
18-Month-Olds Predict Specific Action Mistakes Through Attribution of False Belief, Not Ignorance, and Intervene Accordingly
}

\author{
Birgit Knudsen \\ Max Planck Research Group Communication Before Language \\ Max-Planck-Institute for Psycholinguistics \\ Ulf Liszkowski \\ Max Planck Research Group Communication Before Language \\ Max-Planck-Institute for Psycholinguistics \\ Donders Institute for Brain, Cognition and Behaviour \\ Radboud University
}

\begin{abstract}
This study employed a new "anticipatory intervening" paradigm to tease apart false belief and ignorance-based interpretations of 18-month-olds' helpful informing. We investigated in three experiments whether 18-month-old infants inform an adult selectively about one of the two locations depending on the adult's belief about which of the two locations held her toy. In experiments 1 and 2 , the adult falsely believed that one of the locations held her toy. In experiment 3 , the adult was ignorant about which of the two locations held her toy. In all cases, however, the toy had been removed from the locations and the locations contained instead materials which the adult wanted to avoid. In experiments 1 and 2, infants spontaneously and selectively informed the adult about the aversive material in the location the adult falsely believed to hold her toy. In contrast, in experiment 3 , infants informed the ignorant adult about both locations equally. Results reveal that infants expected the adult to commit a specific action mistake when she held a false belief, but not when she was
\end{abstract}

Correspondence should be sent to Birgit Knudsen, Max Planck Institute for Psycholinguistics, Wundtlaan 1, 6525 XD Nijmegen, The Netherlands. E-mail: Birgit.Knudsen@mpi.nl 
ignorant. Further, infants were motivated to intervene proactively. Findings reveal a predictive action-based usage of "theory-of-mind" skills at 18 months of age.

Recent action-based studies suggest that infants in their second year of life use false belief understanding to guide their social actions. Findings show that infants interact differently with an adult who is misinformed about the referent of an interaction compared with an adult who is accurately informed. For example, in one study, 17-month-old infants were first trained to retrieve an object from one of the two boxes to which an adult pointed (Southgate, Chevallier, \& Csibra, 2010). Subsequently, the objects in the boxes were swapped. In a true belief condition, the adult saw the swap. At test, infants retrieved the object from the box to which the adult pointed. However, in a false belief condition, the adult did not witness that the objects in the boxes were swapped. When the adult now pointed to one of the boxes to request an object, infants retrieved the object from the opposite box. Thus, infants apparently appealed to the adult's false belief and inferred that she wanted the object that was currently in the other box (for a similar paradigm with slightly older, verbal children, see Carpenter, Call, \& Tomasello, 2002).

Similarly, in another study, 18-month-old infants were first trained to help an adult open one of the two boxes (Buttelmann, Carpenter, \& Tomasello, 2009). Subsequently, in a false belief condition, the adult had not witnessed that someone had switched a toy from one to the other box. When the adult then attempted to open the now-empty box, infants opened the opposite box for the adult. However, in a true belief condition, when the adult had seen the switch and now tried to open the empty box, infants helped him to open that box. Thus, depending on what the adult had seen previously, infants apparently attributed different goals to her (respectively, either the goal to retrieve a toy from a box or to open an empty box). The two studies suggest that infants consider an adult's epistemic state when reacting socially. However, the studies also raise important questions regarding the usage and the kind of mental attributions infants make when acting socially.

One question is whether infants act helpfully and appropriately not only in reaction to but also in anticipation of an adult's mistaken action. Predicting others' action mistakes and intervening accordingly is a key skill of efficient and helpful collaborative interaction and communication. The aforementioned action-based studies follow the "explanation"-type structure of classical theory-of-mind tasks ("Why is she pointing to/acting on that box?"). Relatively less is known about infants' active and helpful intervening in "prediction"-tasks ("Where will she search for her toy?"). Two 
recent action-based paradigms suggest that 12- to 18 -month-olds infants help a misinformed adult by communicating to her relevant information before she will act mistakenly. In one study, 18-month-old infants corrected an adult in anticipation of her searching for an object in a wrong location (Knudsen \& Liszkowski, in press). In the experiment, infants watched as an adult searched for an object and found it in the last of four containers. In her absence, the object was then moved to the first container. At test, when the adult re-entered the room, infants spontaneously helped her find the object by pointing to the object's new location. In contrast, infants did not inform the adult in control conditions, when she had either seen the transfer of the object or when it had not been her intention to find the object. In a second study, Knudsen and Liszkowski (2010) found that 12- and 18month-olds warned an adult in anticipation of her acting toward an undesired outcome. In the experiment, infants watched as an adult pretended to hurt herself on an object and subsequently avoid it. In her absence, the object was then moved into her way. At test, when the adult re-entered the room, infants spontaneously pointed to the object the adult intended to avoid. Infants pointed significantly less in control conditions, when the adult had witnessed its reappearance, or when the object had previously caused no harm and the adult did not intend to avoid it. These two studies, thus, suggest that infants in their second year use mental state attributions about others' intentions and epistemic states not only when reacting to others actions but also to intervene proactively and spare others mistakes or harm.

However, a second question is what kind of epistemic states infants attribute. It has been argued that infants simply attribute ignorance - the lack of knowledge - rather than a false representation of reality. For example, in the experiments, infants may have realized that the experimenter did not witness the transfer of an object, and therefore did not know where it was (as opposed to believing it was in the wrong location). They may have then applied a rule of the kind "Doesn't know - gets it wrong" and inferred the correct response without attributing false belief (Fabricius \& Khalil, 2003; Garnham \& Ruffman, 2001; Ruffman, 1996). Looking-time studies, which measure reactions to action outcomes (e.g., Keen, 2003), however, suggest that this is not the case. Eighteen-month-old infants apparently differentiate between false belief and ignorance, as indicated by longer looking times to an agent who has a false belief but acts correctly compared with an agent who is ignorant but acts correctly (Scott \& Baillargeon, 2009; Scott, Baillargeon, Song, \& Leslie, 2010). However, for action-based explanation-type tasks, the alternative explanation is still troublesome, as one may help an adult for both reasons, either because of her lack of knowledge or because of her misguided assumption about reality. 
Action-based prediction tasks can in principle be solved in this alternative way too. Another nonverbal prediction task has employed visual anticipation measures. Southgate, Senju, and Csibra (2007) trained 25-month-olds to make anticipatory gaze shifts to one of the two windows through which an adult would make a reach to obtain a toy from an opaque box in front of that window. Subsequently, the adult did not witness that the object was switched from one to the other box, and thereafter removed from the scene all together. The finding was that infants anticipated a reach through the window where the adult falsely believed the object to be (i.e., where she had last seen it). The study suggests that 2-year-olds attributed false belief and not ignorance, because the desired object had been removed all together from the scene and so the locations to which the actor could potentially make a reach were technically both wrong. Had infants expected the actor to simply "get it wrong," they should have expected her to reach in either location. This was not the obtained pattern, thus making an ignorancebased interpretation less likely. On the other hand, the study did not administer an ignorance condition to compare performance under such conditions more directly. Either way, current action-based studies of both the "explanation"-type paradigm (Buttelmann et al., 2009; Southgate et al., 2010) and the new "anticipatory intervening" paradigm (Knudsen \& Liszkowski, 2010 , in press) are still amenable to an ignorance-based interpretation.

In this study, we sought to tease apart the ignorance and false belief interpretations in a predictive action-based paradigm. We employed a paradigm that combined the logic of the anticipatory looking study by Southgate et al. (2007) and the action-based measure of anticipatory intervening previously used by Knudsen and Liszkowski (2010, in press). In three experiments, we tested whether 18-month-old infants would expect an adult to approach one of the two boxes, depending on her belief about it containing a desired toy. In experiment 1 , an adult falsely believed that her desired object was in one of the two boxes, when in reality it had been removed from the scene all together (as in Southgate et al., 2007). In addition, both boxes were "contaminated" with aversive, to-be-avoided material (as in Knudsen \& Liszkowski, 2010). Following Knudsen and Liszkowski (2010), we expected infants to intervene when appropriate and try to spare the adult the mishap of reaching into the aversive material by pointing it out to her in advance. Based on the recent findings that infants' looking times differentiate between false belief and ignorance-based actions (Scott \& Baillargeon, 2009; Scott et al., 2010), infants should attribute a false belief to the adult and expect her to approach the box that she falsely believed to contain her desired toy. Thus, we predicted that infants would inform the adult selectively about the aversive material in that location (e.g., to warn her appropriately). Experiment 2 addressed procedural aspects and controlled for possible alternative 
explanations of experiment 1 . To confirm that infants did not use ignorance attributions in experiments 1 and 2, we tested infants' performance in an ignorance condition in experiment 3. Experiment 3 used the same paradigm as experiments $1 \& 2$, except that the adult was ignorant about which of the two boxes held her object. Consequently, if infants correctly attributed ignorance to the adult (Scott \& Baillargeon, 2009; Scott et al., 2010), they should expect her to approach either box by chance and inform her about the aversive material in both locations equally.

\section{EXPERIMENT 1}

\section{Method}

\section{Participants}

Infants were recruited from a database of parents who had volunteered to participate in infancy research. Nineteen 18-month-olds (eight boys; $M$ age $=18 ; 12$, range $=17 ; 26-18 ; 27$ ) participated in the study. Five additional infants had to be excluded from analyses, because of fussiness (2), parental interference (2), or technical failure (1). Eight individual trials of seven infants had to be discarded, because of parental interference (2), experimenter error (1), technical failure (1), or fussiness (4).

\section{Set-up}

Infants sat on their parents' lap at the long side of a table. The table was centered in the back of the testing room. Infants faced the main experimenter (E1) who sat across the infant at the table. Behind E1, approximately $1 \mathrm{~m}$ to her left and right, stood two square pillars on the floor (about $1 \mathrm{~m}$ high, $34 \mathrm{~cm}$ wide). Behind E1's chair, a straight path was roped-off with four posts $(0.5 \mathrm{~m}$ high). The path diverged after about $2 \mathrm{~m}$ in a T-shape to the left and to the right of the room (approximately another $2 \mathrm{~m}$ ). The left path led behind a cupboard ( $2 \mathrm{~m}$ high), and the right path led to the door of the room. Both sides served as entrances to the scene. About $1.5 \mathrm{~m}$ to the infant's left, perpendicular to the table's long side, stood a 2-m long room divider (1.45 $\mathrm{m}$ high). Behind the room divider hid a second experimenter (E2), watching the procedure on a video screen that was connected to the cameras which provided a full view on the scene. There were five cameras in total. Two cameras were positioned to the far left and right corners on the infant's side, recording the experimenter and the room behind her. A spy camera was attached to the ceiling above the table providing a bird's eye view on the infant and the table. Two additional cameras were each hidden in the pillars left and right from E1, providing a close-up view on the infant. 


\section{Materials}

There were four different sets of three identical containers. Each set was used in one of the four trials. All containers had an opening on the front side, big enough to reach in. Two containers of each set were hidden inside the left and right pillars, and the third container was hidden under the table. Each of the containers under the table contained one toy: (a) a marble, (b) a plastic duck, (c) a ladybug, (d) a spring with a spinning top, and a silver ball attached. Each of the four containers under the table also contained objects marked as aversive: (a) gray crumpled-up tissues, (b) green wads of elastics, (c) pieces of crumpled, red-grayish plastic nets, and (d) green crumpled-up tissues. Four additional, identically looking aversive objects were hidden behind the top of the right pillar out of infants' sight.

\section{Procedure}

There were four trials with a brief play break after two trials. Each trial consisted of four phases: demonstration, epistemic induction, switch, and test. Parents were instructed to remain silent during testing and not to interfere with the procedure.

\section{Demonstration}

E1 placed an empty container from inside each pillar on top of each pillar. The opening of the containers faced the infant. Then she sat down at the table and placed the corresponding container of that set from under the table on the table in front of her, with the opening facing the infant. The infant- but not she - could see the toy and the aversive object inside the container. She said: "Let's play with this." She reached from behind into the front opening of the container and bumped into the aversive object (see Figure 1, photograph 1). She emoted with facial expressions of disgust, saying: "Yak! What is this?" She pushed the object to the left side of the table, saying with the same facial expression: "Yak, this is disgusting! I have to clean this up." She picked up the object with her thumb and index finger, and put it behind the container on top of the left pillar, out of the infant's sight (see Figure 1, photograph 2). Then she reached again into the container on the table. This time she grabbed the toy. She said "Hey, look at this!" and began to playfully insert and then retrieve the toy from the container, saying "Now it's in there....now I'll get it out again" (see Figure 1, photograph 3).

Next, E1 took the toy out of the container and removed the container under the table. She turned to one of the two containers on the pillars and playfully inserted and retrieved the object in the same manner as before. 
678 KNUDSEN \& LISZKOWSKI

(a) Demonstration

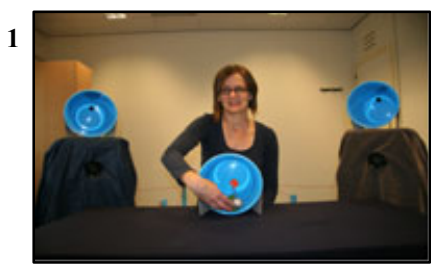

2

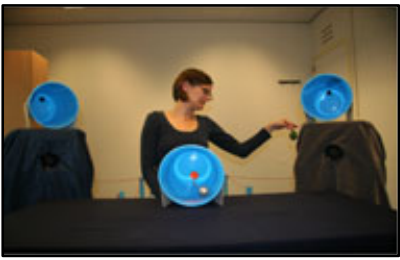

3

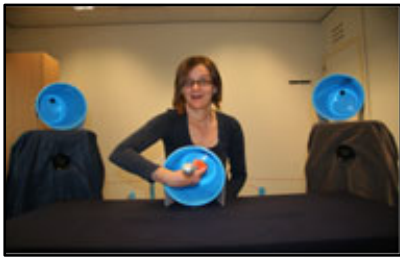

4

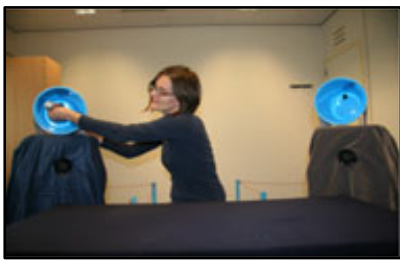

5

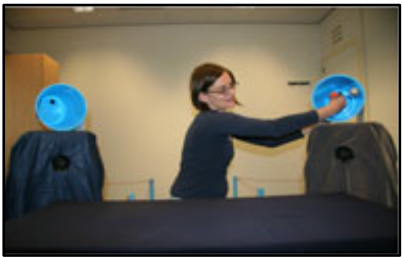

Epistemic induction

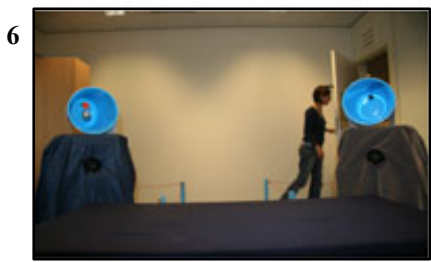

(b)

Switch

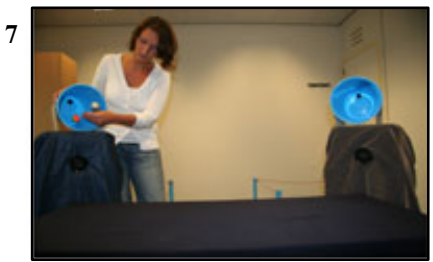

8

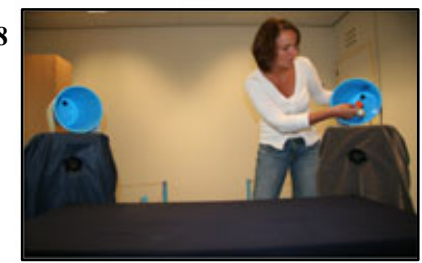

9

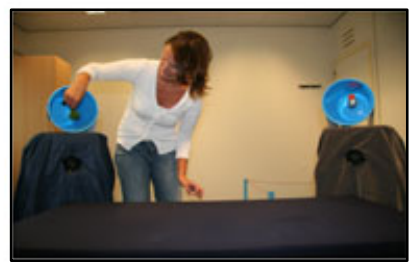

10

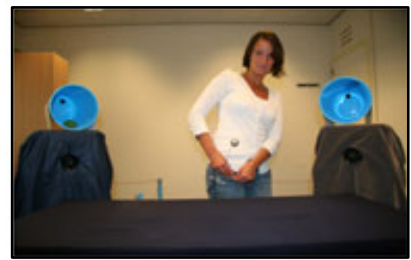

11

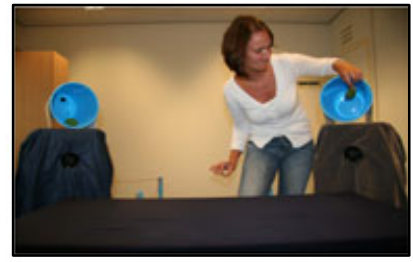

Test

12

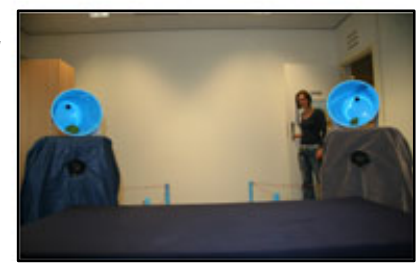


Figure 1 Staged photographs of the general procedure of experiments 1, 2, and 3. (a) Demonstration: 1. E1 unintentionally bumps into aversive material. 2. E1 removes the aversive material behind the right pillar. 3 . E1 plays with the toy. 4 \& 5. E1 plays with the toy alternately at both containers on the pillars. Epistemic induction: 6. E1 has put the toy in one container (target container) and leaves (Note: this step differed in experiment 3). (b) Switch: 7. E2 finds the toy in the target container. 8. E2 puts the toy into the distractor container. 9. E2 has bumped into the aversive material and moves it into the target container. 10. E2 has taken the toy from the distractor container and puts it demonstratively into her pocket. 11. E2 has bumped into the aversive material and moves it into the distractor container. Test: 12. The toy is removed from the scene and both boxes are baited with aversive material. E1 re-enters the room and states her intention to continue playing. She walks a roped-off L-shaped path toward the table.

Then she turned to the other container and played in the same manner (see Figure 1, photographs 4-5). The play sequence on both pillar containers was then repeated, thus totaling four play episodes, two on each of the containers on the pillars. The side where the play began was counterbalanced across participants and alternated in an a-b-b-a design across the four trials. Then E1 interrupted the play saying "Oh, I have to leave." She held up the toy and said "We go on playing with this in a minute, okay?"

\section{Epistemic induction}

This phase served to establish El's belief about the toy's final location. Before leaving, E1 put the toy into one container (target container). This was always the opposite container with which she had played last (following the counterbalancing above; see Figure 1, photograph 6). She said: "Well, I'll leave it here. See, there it is." To equate for this step toward the target container she then turned to the other container (distractor container), adjusted its position by shuffling it around and said "All right." With every comment, E1 alternated gaze with the infant. Subsequently, she left the scene either through the door or by hiding behind the cupboard, saying "Okay, I'll be back in a bit." For half of the infants, El's side of entrance was on the same side as the target container in the first two trials and on the opposite side in the next two trials. The other half got the reverse order.

\section{Switch}

In this phase, E2 removed the toy from the scene all together and baited both containers with the aversive objects E1 wanted to avoid. E2 appeared from behind the room divider, greeted the infant, and started to clean the target container. She accidentally found the toy inside the container, took it out, held it up, and said "Oh. Well, I put it here." She put it into the distrac- 
tor container (see Figure 1, photographs 7-8). Then she turned back to continue cleaning the target container. Accidentally, she bumped into the aversive object behind the container. She emoted with facial expressions of disgust and said: "Yak! What is this?" She picked up the object with her thumb and index finger, placed it on the table, and continued cleaning the container. Then she moved on to clean the table and bumped into the object again. She said with the same facial expression: "Yak, this is disgusting!" and moved the object from the table into the target container (see Figure 1, photograph 9).

Then she turned to the distractor container and the sequence was repeated: She encountered the toy inside the distractor container, took it out, and held it up. She said: "Oh. Well, I take this with me" and put the toy demonstratively into her trouser's pocket (see Figure 1, photograph 10). She continued cleaning the container, bumped into the aversive object behind the container, and emoted disgust. She put the object on the table and continued cleaning the container. Then she turned to clean the table, bumped again into the object, emoted disgust, and moved it from the table into the distractor container (see Figure 1, photograph 11). Then she finished cleaning the table, said with satisfaction "Well, now all is clean," and hid behind the room divider. By the end, E1's toy had, thus, been removed from the scene, and both containers were baited with aversive material.

\section{Test}

E1 came back into the room (see Figure 1, photograph 12). First, she stood still and said "Now we can play again." Then she walked the ropedoff path toward the table $(2 \times 2 \mathrm{~m})$. On two predefined spots (at the T-junction, and half way toward her chair), E1 briefly stopped, looked at the infant, and said "Okay, let's go on." The test phase (about $30 \mathrm{sec}$ ) was over as soon as E1 had sat down. If infants pointed during this period, E1 responded by saying "Yes" and continued her predetermined sequence. All trials ended with $\mathrm{E} 1$ reaching into the target container, bumping into the aversive object, and emoting disgust. Then she removed the containers to get "other ones to play with" for the next trial.

\section{Coding and data analysis}

Pointing was defined as a half or fully extended arm with the open hand palm down or the extended index finger. The arm was extended in the air and toward discernable direction. The arm extension had to have a clear onset and end, with a clear trajectory toward the target or distractor container. The direction could easily be coded from the recording of the bird's 
eye spy camera above the table. Points were coded by an assistant blind to the hypothesis if they occurred during the test phase and were directed to the target or distractor container. A second coder, blind to the hypothesis, recoded a random subset of six infants. Excellent inter-rater reliability was obtained, Cohen's Kappa $=.92$. In addition, to ascertain that E1 did not show in her behavior which container she would approach during the test phase, a third coder, blind to the hypothesis, judged for all infants in a twoalternative forced-choice procedure which container E1 would reach into during the test phases. The third coder was at chance in predicting the location E1 would reach into, Binominal, $p=1$. Analyses were nonparametric and one-tailed when following our planned comparisons.

\section{Results and discussion}

As depicted on the left of Figure 2, infants pointed more often to the target container than the distractor container (mean number of points per trial, .711 vs. .410 , respectively; Wilcoxon signed ranks test, $Z=-2.63$, $p=.004)$. This pattern was stable across infants, with the majority of infants pointing more to the target container than the distractor container $(63.2 \%)$ and only one infant $(5 \%)$ pointing more to the distractor than the target container, Binomial test, $p=.002$. Similarly, infants' mean proportion of trials in which the first point was directed at the target container was higher (Mean Proportion $=.373$ ) than the mean proportion of trials in which the first point was directed at the distractor container (Mean Proportion $=.184$ ), Wilcoxon signed ranks test, $Z=-2.17, p=.02$ (see left side of Figure 3). Thus, infants informed the adult selectively about the aversive object in the container from which she was about to retrieve her toy, supporting the interpretation that infants attributed to the adult a false belief. The possibility that infants attributed only ignorance to the adult and applied the rule "She doesn't know, she gets it wrong" (Garnham \& Ruffman, 2001; Ruffman, 1996), appears thus less likely, because then infants should have pointed randomly to the containers that had both been emptied and now contained an aversive object.

There was no indication that infants learnt this behavior over trials. If any, infants tended to point more to the target in the first half of the experiment (mean number of points per trial $=.84$ ) than in the second half (mean number of points per trial $=.44$ ), Wilcoxon signed ranks test, $Z=-1.69$, $p=.091$, two-tailed. Previous action-based studies (Buttelmann et al., 2009; Southgate et al., 2010) tested performance on a single trial only. However, in those studies, infants were first trained to produce the target behavior, and if they did not, they were excluded from the analyses. In our sample, 11 of the 14 infants who pointed at least once $(79 \%)$ pointed already 


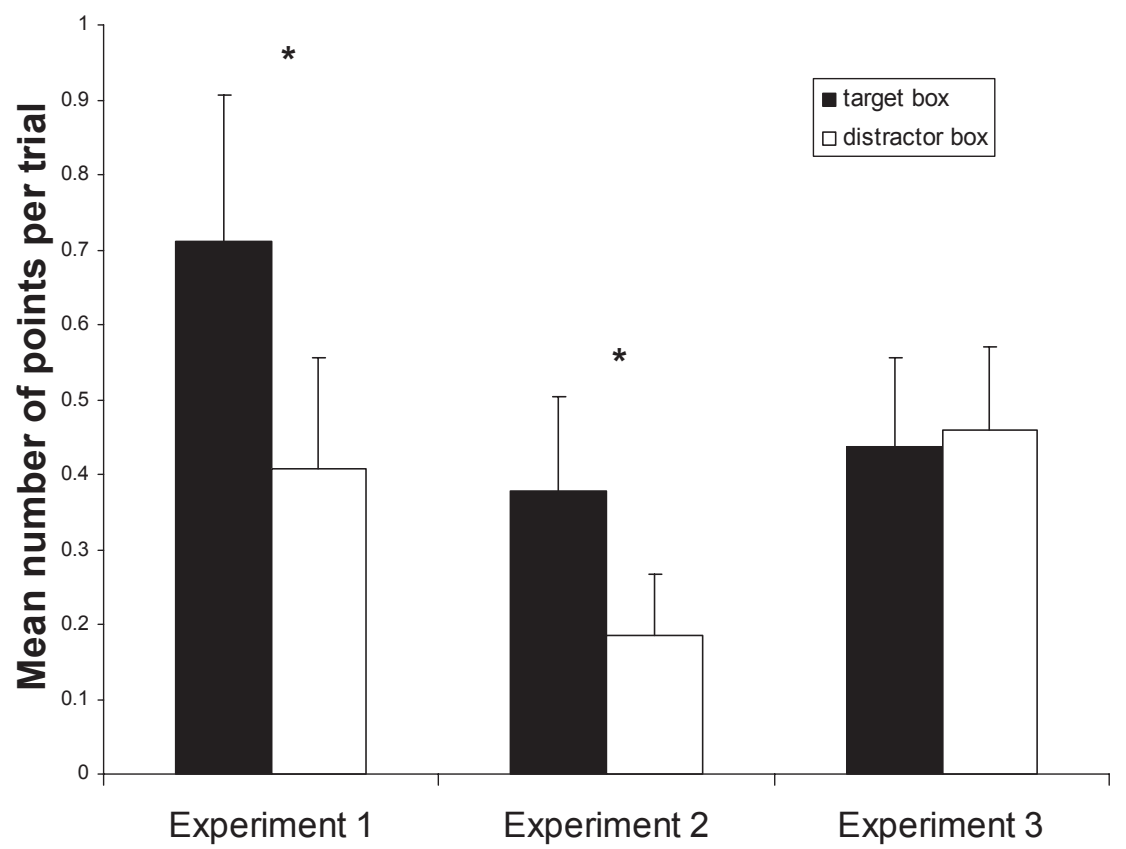

Figure 2 Mean number of points per trial to the target and distractor containers in experiments 1, 2, and 3. Error bars represent the standard errors of the means.

on the first trial. The first point in the first trial was more often directed toward the target container (7/11) than the distractor container $(4 / 11)$, but this did not reach statistical significance (Binominal test, $p=.27$ ). Thus, although there was no learning over trials and the pattern of mean differences was already there on the first trial, the first-trial differences failed to reach statistical significance, presumably due to the reduced power of singletrial analyses and fewer participants.

Additional analyses revealed that infants' pointing to the target container was not biased by the side of the experimenter's entrance, Wilcoxon signed ranks test, $Z=-1.41, p=.158$ (two-tailed; mean number of points per trial, congruent side $=.500$; incongruent side $=.833$ ). Further, the experimental procedure of the epistemic induction excludes the possibility that infants pointed simply to the container E1 last attended, as E1 always last attended to the distractor container before she left the scene. The experimental procedure of the switch excludes also the possibility that infants pointed simply to where they had last seen the toy, because E2 always moved the toy 


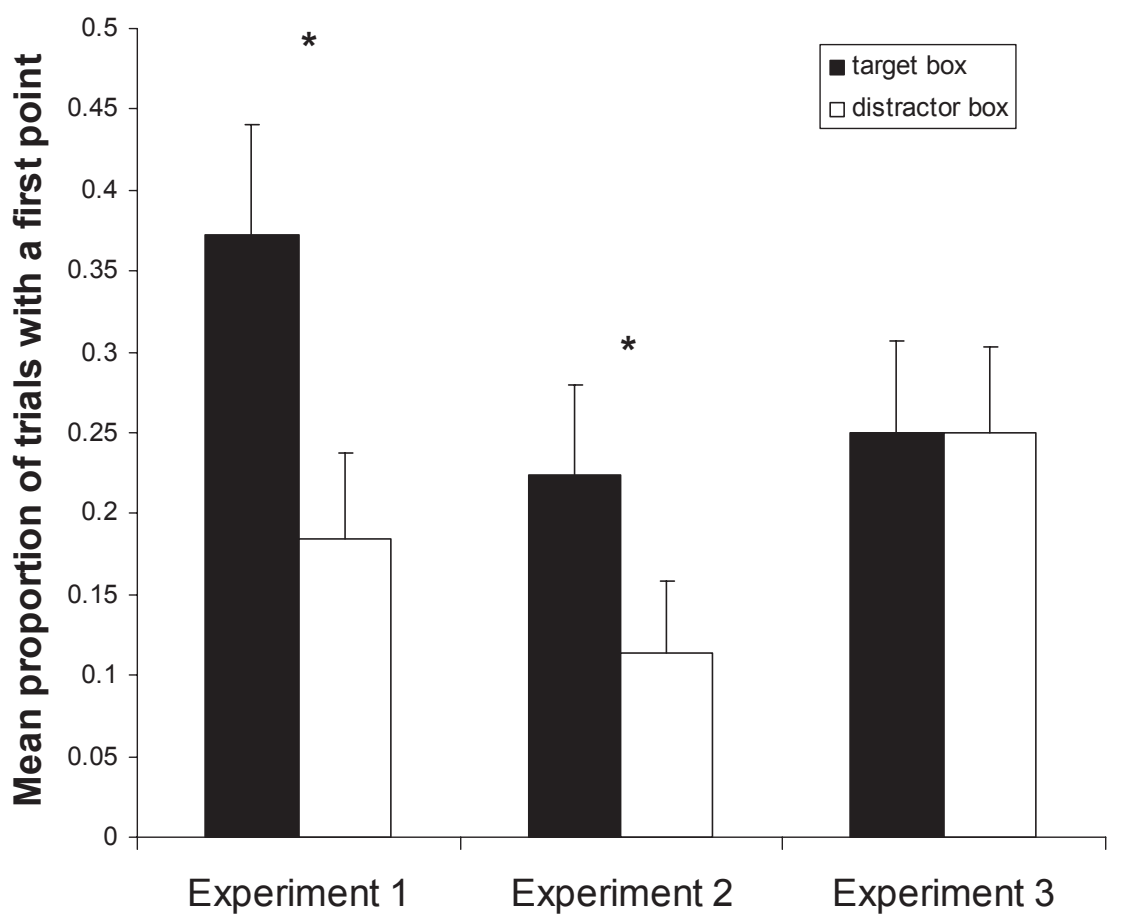

Figure 3 Mean proportion of trials in which a first point was directed to the target or distractor container in experiments 1,2, and 3. Error bars represent the standard errors of the means.

into the distractor container before she removed the toy completely by putting it into her pocket.

However, it might be that infants pointed more to the target container because it was more salient or easier to remember. For example, during the epistemic induction, E1 spent slightly more time acting on the target container when saying "Well, I leave it here. See, there it is" than on the distractor container when saying "All right". This might have rendered the target container more salient to the infant. It is theoretically also possible that infants only paid attention to the first container E1 acted on during the epistemic induction. As this was always the target container, infants might have simply pointed more to it, because they remembered it better. In experiment 2 , we, therefore, aimed at replicating the effect while controlling for these alternative possibilities. 


\section{EXPERIMENT 2}

\section{Method}

\section{Participants}

Nineteen 18-month-olds (10 boys; $M$ age $=18 ; 20$, range $=18 ; 6-18$; 28) participated in the study. Five additional infants had to be excluded from analyses because of fussiness (2), parental interference (1), technical failure (1), or experimenter error (1). Six individual trials of six infants had to be discarded, because of experimenter error (2) or fussiness (4).

\section{Set-up and materials}

The set-up and the materials were exactly the same as in experiment 1.

\section{Procedure}

The procedure was identical to that in experiment 1 except for the two aspects during the epistemic induction. First, E1 now acted the same toward each container and the sentences were equated for content, length, and intonation: In the epistemic induction, E1 put the toy into the target container and said "Well, I leave it here. See, there it is." Regarding the distractor container, E1 reached into the distractor container and said "Well, I leave this one empty. See it is empty." Second, the order in which E1 acted on the containers (target container first vs. distractor container first) was counterbalanced. E1 acted on two consecutive trials either first on the target container or on the distractor container. The order was counterbalanced across infants. As in experiment 1, E1 left the scene by saying "Okay, I'll be back in a bit." Because of a slight imbalance in the design, for eight infants, E1's side of entrance was always congruent with the side of the target container, but this did not affect the results, and the previous experiment had revealed no side bias.

\section{Coding and data analysis}

The coding and analyses were exactly the same as in experiment 1 . Inter-rater reliability on infants' number of points to either location was excellent for a randomly chosen six infants, Cohen's Kappa $=.80$. The third coder was at chance in predicting the location E1 would reach into in all test phases of all infants tested (one test phase of one trial could not be coded because of failure of the cameras directed toward E1), Binominal, $p=.282$. 


\section{Results and discussion}

Experiment 2 replicated the main finding of experiment 1 . As depicted in the middle of Figure 2, infants pointed more often to the target container than the distractor container (respectively, mean number of points per trial, .377 vs. .184, Wilcoxon signed ranks test, $Z=-1.89, p=.03)$. This pattern was stable across infants, with the majority of infants $(47.4 \%)$ pointing more to the target container than the distractor container, and less infants pointing more to the distractor than the target container (10.5\%), Binomial test, $p=.033$. Similarly, infants' proportion of trials in which the first point was directed at the target container (Mean Proportion $=.224$ ) was higher than the proportion of trials in which the first point was directed at the distractor container (Mean Proportion $=.114)$, Wilcoxon signed ranks test, $Z=-1.80, p=.036$ (see middle panel of Figure 3). Thus, infants informed the adult selectively about the aversive object in the container from which she was about to retrieve her toy, even when the saliencies of the containers were controlled. Also the order of the containers on which E1 had acted first during the epistemic induction did not make a significant difference, neither with regard to the mean number of points to the target container, Wilcoxon signed ranks test, $Z=-.99, p=.321$, nor with regard to the number of infants that pointed at least once to the target container, McNemar, $p=.774$. Thus, infants did not attend to or remembered the target container better when it was acted on first or second.

As before, there was also no indication that infants learnt the target behavior over trials. Infants tended to point to the target equally often in the first and in the second half of the experiment (respectively, mean number of points per trial .32 vs. .45), Wilcoxon signed ranks test, $Z=-.79$, $p=.427$. Eight of the 11 infants who pointed at least once $(73 \%)$ pointed on the first trial. The first point in the first trial was more often directed toward the target container $(5 / 8)$ than the distractor container $(3 / 8)$, but this did not reach statistical significance, Binominal test, $p=.36$. Additional analyses revealed that infants' pointing to the target container was not biased by the side of the experimenter's entrance, (mean number of points per trial, same side $=.500$ vs. different side $=.591$, Wilcoxon signed ranks test, $Z=-.33, p=.739$ ). Infants in experiment 2 pointed overall slightly less compared with experiment 1 , possibly reflecting individual differences in the spontaneous use of pointing. However, a direct comparison on the mean number of points across the two experiments revealed no significant differences, Mann-Whitney $U$ test, $Z=-1.53, p=.138$, two-tailed.

Thus, in two experiments, infants informed the adult selectively about an aversive object in the container they expected her to act on according to her false belief. Had they expected the adult to have no representation of the 
object's location whatsoever, infants should have guessed at chance which container she would approach. That is, if infants had intervened based on attributing ignorance instead of false belief, they should have informed the adult about the aversive materials in both containers equally. To test this prediction directly, we administered an ignorance condition in experiment 3 .

\section{EXPERIMENT 3}

In experiment 3, we tested whether infants would correctly attribute ignorance to an adult who did not know in which of the two containers her toy was and whether, in contrast to the false belief conditions, infants would inform the adult about the aversive objects in both locations equally.

\section{Method}

\section{Participants}

Nineteen 18-month-olds (10 boys; $M$ age $=18 ; 20$, range $=18 ; 7-18$; 27) participated in the study. Five additional infants had to be excluded from analyses, because of fussiness (3) or experimenter error (2). Thirteen individual trials of 11 infants had to be discarded, because of experimenter error (5) or fussiness (8).

\section{Set-up and materials}

The set-up and the materials were exactly the same as in experiments 1 and 2 .

\section{Procedure}

The procedure was identical to that in experiment 2 except for a minimal change during the epistemic induction. After E1 put the toy into one container (henceforward also called target container) and said "Well, I leave it here. See, there it is" she simply took the toy out of the target container again. As in the previous experiments, she then turned to the other container (henceforward also called distractor container), reached into it, and said "Well, I leave this one empty. See it is empty." The order was counterbalanced as in experiment 2. Subsequently, she stood up and said as in experiments 1 and 2 "Okay, I'll be back in a bit". At that moment, E2 appeared from behind the room divider, and E1 and E2 greeted each other friendly. E1 handed E2 the toy, clearly visible to the infant, and said again to the 
infant "I'll be back in a bit" and left the scene. The switch started with E2 putting the toy into the target container and continued as in experiments 1 $\& 2$.

\section{Coding and data analysis}

The coding and analyses were exactly the same as in experiments 1 and 2. Inter-rater reliability on infants' number of points was excellent for a randomly chosen 6 infants, Cohen's Kappa $=.84$. The third coder was at chance in predicting the location E1 would reach into in all test phases of all infants tested, Binominal, $p=.45$.

\section{Results and discussion}

As depicted on the right Figure 2, infants pointed equally often to the target container and the distractor container (respectively, mean number of points per trial .439 vs. .461, Wilcoxon signed ranks test, $Z=-.21, p=.838$ ). This pattern was stable across infants, with most infants pointing equally much to both containers $(47.3 \%)$, and about the same number of infants pointing more to the target than the distractor container $(21.1 \%)$ or more to the distractor than the target container (31.6\%), Binomial test, $p=.754$. Similarly, infants' first points were equally likely to be directed to the target container (mean proportion of trials with a first point to the target $=.25$ ) or to the distractor container (mean proportion of trials with a first point to the distractor $=.25$ ), Wilcoxon signed ranks test, $Z=-.28, p=.778$ (see left panel of Figure 3). Thus, infants indeed expected the adult to reach into either container to retrieve the toy and informed her correspondingly about the aversive objects in both containers equally.

When looking only at the first trial, 79\% (11/14) of the pointing infants pointed on that trial. The first point in the first trial was equally often directed toward the target container $(5 / 10)$ or the distractor container $(5 / 10)$, Binominal test, $p=1$, and one infant pointed with both arms to both containers at the same time. Additional analyses revealed that infants' pointing to the target container was not biased by the side of the experimenter's entrance, Wilcoxon signed ranks test, $Z=0$, $p=1$ (two-tailed; mean number of points per trial to congruent side $=.441$ vs. incongruent side $=.471$ ), and the order of containers on which E1 acted first did not make a significant difference, neither with regard to the mean number of points per trial to the target container, Wilcoxon signed ranks test, $Z=-1.19, p=.233$, nor with regard to the number of infants that pointed at least once to the target container, McNemar, $p=.375$. 


\section{GENERAL DISCUSSION}

The current study teased apart false belief and ignorance-based interpretations of infants' prospective informing in an action-based anticipation paradigm. In experiments 1 and 2, infants informed an adult selectively about the container which the adult falsely believed held her toy. Importantly, the procedure excluded a "pull of the real," as the toy had been removed from the scene all together (see Southgate et al., 2007). Therefore, it is unlikely that infants expected the adult to make a mistake because of her lack of knowledge - as both locations were wrong. Thus, if infants would have attributed only ignorance or followed the rule "doesn't know - gets it wrong" (Garnham \& Ruffman, 2001; Ruffman, 1996), they should have informed the adult about both containers equally. As experiment 3 showed, infants indeed informed the adult about both containers equally, but only when the adult was ignorant about the location of her toy - not when she had a false belief about its location. The specific procedures of the three experiments excluded also several other lower level explanations, which invoke the adult's or infant's first or last place of attention, the presence of the toy, or other saliency or order effects. The findings, thus, reveal that infants expect an adult to make a specific mistake when the adult has an incorrect representation of reality - they inform her selectively; and that infants expect the adult to act at chance when she is ignorant- - they inform her globally.

The current study further shows that infants were motivated to intervene spontaneously and make active use of their social understanding by communicating. Infants did not have to learn a behavioral response in advance, as the adult never pointed or engaged the infant in pointing before the experiment. Infants also did not learn the behavior over trials, as the comparison of the first and second half of the experiment showed. However, not every infant pointed on the first trial. At first, this may seem at odds with the action-based helping study by Buttelmann et al. (2009), in which infants helped on a single first trial. However, it is important to recall that that study excluded infants who did not show the response behavior. In addition, in that study, some of the infants reacted only after a prompt from another person. When taking this into account, the percentages of spontaneous first trial helping become comparable between the studies. However, it is also possible that infants more readily react to a mistaken action than that they intervene proactively before a mistaken action has happened. Finally, in contrast to previous studies (Buttelmann et al., 2009; Southgate et al., 2007, 2010), infants in the current paradigm were not familiarized or trained on the response measure beforehand. Thus, although our study shows that not all infants were equally motivated to point spontaneously on the very first 
trial, and that there was some indication of individual differences across experiments 1 and 2, it is still a remarkable demonstration of spontaneous communication in a staged laboratory setting.

With regard to the cognitive underpinnings of infants' success on recent theory-of-mind tasks, the current debate centers around the question as to whether the obtained results best fit a mentalistic interpretation or a behavioral rule interpretation. Do infants understand that the mind mediates between perception and action or do they instead follow behavioral rules that link one behavior to another? Preschool children have a mentalistic ("representational") theory-of-mind, as they can engage in and verbalize their mentalistic reasoning even in the absence of behavioral situations that would provoke a reaction (Perner, 2010). In contrast, there is no such evidence for prelinguistic infants, and so Perner (2010) has argued that theoryof-mind tests in infancy are most parsimoniously explained by the use of behavioral rules. One problem with the latter account is that it is somewhat unclear what these behavioral rules are, especially as they are often based on post hoc interpretations of specific task performances (e.g., Perner \& Ruffman, 2005). Some of the previously formulated rules (e.g., "three-way-association," Perner \& Ruffman, 2005; "Doesn't know- gets it wrong," Ruffman, 1996) have also been empirically refuted (Scott \& Baillargeon, 2009; Scott et al., 2010; Träuble, Marinović, \& Pauen, 2010). In Perner's (2010) more recent and general formulation, behavioral rules "capture the workings of the mind without mentioning the mind" (Perner, 2010, p. 257). In this case, mentalistic and behavioral rule accounts make the same predictions about theory-of-mind performance. Before being able to mention the mind, infants may, thus, have an implicit or practical "theory-of-mind." However, it is currently debatable how postulating two systems may be more parsimonious than assuming one and what the developmental antecedents and consequences for each system could be (e.g., Apperly \& Butterfill, 2009).

The current study demonstrates that infants have the skills of anticipating another's belief-specific action mistake before it is apparent in the overt behavior and that infants are motivated to intervene prospectively through communication. The study is in line with recently accumulating evidence from other infant "theory-of-mind" studies (for recent reviews see Baillargeon, Scott, \& He, 2010; Caron, 2009) and cannot easily be interpreted in terms of the specific behavioral rule that adults who lack information will commit a specific action mistake (Ruffman, 1996). Thus, already before they have acquired language in earnest, infants actively use their theory-of-mind skills to communicate prospectively. It will be important to unravel whether the underlying cognitive format remains the same over development, whether it is supplanted or complemented by another system, and what the underlying nature of prelinguistic and linguistic social cognitive representations are. 


\section{ACKNOWLEDGMENTS}

We like to thank Loraen Kaltenschnee, Margret van Beuningen, Marloes van der Goot, Stephanie Voigt, Merel Fokkema and Mireille Hassemer for their assistance with data collection, and Ludy Cilissen, Bas Roset and Nick Wood for their technical support.

\section{REFERENCES}

Apperly, I.A., \& Butterfill, S.A. (2009). Do humans have two systems to track beliefs and belieflike states? Psychological Review, 116(4), 953-970.

Baillargeon, R., Scott, R. M., \& He, Z. (2010). False-belief understanding in infants. Trends in Cognitive Sciences, 14, 110-118.

Buttelmann, D., Carpenter, M., \& Tomasello, M. (2009). Eighteen-month-old infants show false belief understanding in an active helping paradigm. Cognition, 112, 337-342.

Caron, A. J. (2009). Comprehension of the representational mind in infancy. Developmental Review, 29, 69-95.

Carpenter, M., Call, J., \& Tomasello, M. (2002). A new false belief test for 36-month-olds. The British Journal of Developmental Psychology, 20, 393-420.

Fabricius, W. V., \& Khalil, S. L. (2003). False beliefs or false positives? Limits on children's understanding of mental representation. Journal of Cognition and Development, 4(3), 239262.

Garnham, W. A., \& Ruffman, T. (2001). Doesn't see, doesn't know: Is anticipatory looking really related to understanding of belief? Developmental Science, 4(1), 94-100.

Keen, R. (2003). Representation of objects and events: Why do infants look so smart and toddlers look so dumb? Current Directions in Psychological Science, 12, 79-83.

Knudsen, B., \& Liszkowski, U. (2010, March). 18-month-old infants warn others in anticipation of negative action effects. Poster presented at 17th Biennial International Conference on Infant Studies, Baltimore, $M D$.

Knudsen, B., \& Liszkowski, U. (in press). Eighteen- and 24-month-old infants correct others in anticipation of action mistakes. Developmental Science, doi: 10.1111/j.1467-7687.2011. 01098.x.

Perner, J. (2010). Who took the cog out of cognitive science? Mentalism in an era of anticognitivism. In P. A. Frensch \& R. Schwarzer (Eds.), Cognition and neuropsychology: International perspectives on psychological science (Vol. 1, pp. 241-261). Hove, UK: Psychology Press.

Perner, J., \& Ruffman, T. (2005). Infants' insight into the mind: How deep? Science, 308, 214 216.

Ruffman, T. (1996). Do children understand the mind by means of simulation or a theory? Evidence from their understanding of inference. Mind \& Language, 11(4), 388-414.

Scott, R. M., \& Baillargeon, R. (2009). Which penguin is this? Attributing false beliefs about object identity at 18 months. Child Development, 80(4), 1172-1196.

Scott, R. M., Baillargeon, R., Song, H., \& Leslie, A.M. (2010). Attributing false beliefs about non-obvious properties at 18 months. Cognitive Psychology, 61, 366-395.

Southgate, V., Chevallier, C., \& Csibra, G. (2010). Seventeen-month-olds appeal to false beliefs to interpret others' referential communication. Developmental Science, 13(6), 907-912. 
Southgate, V., Senju, A., \& Csibra, G. (2007). Action anticipation through attribution of false belief by 2-year-olds. Psychological Science, 18, 587-592.

Träuble, B., Marinović, V., \& Pauen, S. (2010). Early theory of mind competencies: Do infants understand others' beliefs? Infancy, 15, 434-444. 